\title{
The phylogenetic conundrum of Lutzia (Diptera: Culicidae: Culicini): a cautionary account of conflict and support
}

\author{
Ian J. Kitching, C. Lorna Culverwell and Ralph E. Harbach ${ }^{*}$ \\ Department of Life Sciences, Natural History Museum, \\ Cromwell Road, London SW7 5BD, UK \\ *Corresponding author, e-mail: r.harbach@nhm.ac.uk \\ Published online 12 May 2014; published online 10 June 2015
}

\begin{abstract}
Lutzia Theobald was reduced to a subgenus of Culex in 1932 and was treated as such until it was restored to its original generic status in 2003, based mainly on modifications of the larvae for predation. Previous phylogenetic studies based on morphological and molecular data have provided conflicting support for the generic status of Lutzia: analyses of morphological data support the generic status whereas analyses based on DNA sequences do not. Our previous phylogenetic analyses of Culicini (based on 169 morphological characters and 86 species representing the four genera and 26 subgenera of Culicini, most informal group taxa of subgenus Culex and five outgroup species from other tribes) seemed to indicate a conflict between adult and larval morphological data. Hence, we conducted a series of comparative and data exclusion analyses to determine whether the alternative positions of Lutzia are due to conflicting signal or to a lack of strong signal. We found that separate and combined analyses of adult and larval data support different patterns of relationships between Lutzia and other Culicini. However, the majority of conflicting clades are poorly supported and once these are removed from consideration, most of the topological disparity disappears, along with much of the resolution, suggesting that morphology alone does not have sufficiently strong signal to resolve the position of Lutzia. We critically examine the results of other phylogenetic studies of culicinine relationships and conclude that no morphological or molecular data set analysed in any study conducted to date has adequate signal to place Lutzia unequivocally with regard to other taxa in Culicini. Phylogenetic relationships observed thus far suggest that Lutzia is placed within Culex but further data and extended taxon sampling are required to confirm its position relative to Culex.
\end{abstract}

\section{Keywords}

Culicomorpha; morphology; phylogeny; systematics; taxonomy

\section{Introduction}

Culicini (Diptera: Culicidae: Culicinae), the second largest tribe of mosquitoes, includes 795 currently recognised species classified into four genera: Culex Linnaeus, the largest, has 768 species, Deinocerites Theobald has 18 species, Galindomyia Stone \& Barreto one species and Lutzia Theobald has eight species. Lutzia was established by 
Theobald (1903) as a distinct genus for the Neotropical species Culex bigoti Bellardi. Edwards (1932) reduced Lutzia to a subgenus of Culex and subsequent authors accepted this subgeneric status. Belkin (1962a) surmised that "Lutzia is a very ancient derivative" of Culex, with apparently strongest affinities with subgenus Culex. Although Tanaka et al. (1979) treated Lutzia as a subgenus, they felt it was "more reasonable to consider Lutzia as a genus" because it was morphologically more distinct than other subgenera of Culex. Acting on this suggestion, Tanaka (2003) formally restored Lutzia to its original generic status.

Species of Lutzia occur in the Afrotropical, Oriental, southern Palaearctic, Australasian and Neotropical regions. The adults are large mosquitoes, larger than species of genus Culex. The larvae are predaceous, principally on larvae of other mosquito species. The immature stages predominantly inhabit ground-water habitats, but are occasionally found in phytotelm and artificial containers. Females attack domestic animals and sometimes humans, but otherwise very little is known of the bionomics of the adults.

Genus Lutzia is based mainly on the modifications of the larvae for predation; few characters of the adults are regarded as sufficiently distinctive to justify the separation of the group from genus Culex. The maxillary palpi of males are longer than the proboscis with the distal two palpomeres upturned and setose (except in Lt. shinonagai (Tanaka, Mizusawa \& Saugstad) of subgenus Insulalutzia); the palpi of females are no more than one quarter the length of the proboscis. The lower mesepimeral setae are long and four to eight in number (usually one, but infrequently 2-4 and rarely 5, are present in Culex; CLC \& REH, unpublished data). The anterior surfaces of the femora and tibiae of the fore- and midlegs are mottled with patches of pale scales. The gonocoxite of the male genitalia lacks scales, the subapical lobe bears three prominent setae (setae $a-c$ ) but lacks a foliform seta (seta $g$ ), and the phallosome is simple, formed of a single pair of lateral sclerites (as in species of subgenus Culex, lateral and aedeagal sclerites are not distinguished). The mouthparts of the larvae are highly modified for predation. The lateral palatal brushes consist of about 40 stout and strongly pectinate elements and the mandibles have large teeth for capturing and holding prey. The siphon is short with a continuous mid-posterior row of setae, and the pecten usually extends the whole length of the siphon (except in Lt. shinonagai where is it confined to mid-length). The sclerotized dorsal surface of the terminal segment (segment $\mathrm{X}$ ) is strongly extended caudad, making the segment appear long and pointed.

A number of published and unpublished phylogenetic studies based on morphological and molecular data support the monophyly of all of the generic-level groups of Culicini except subgenera Culex, Eumelanomyia Theobald and Neoculex Dyar (Mallampalli 1995; Miller et al. 1996; Isoe 2000; Navarro \& Liria 2000; Juthayothin 2004; St John 2007; Deus 2009; Vesgueiro et al. 2011; Demari-Silva et al. 2011; Harbach et al. 2012). However, despite this agreement, and bearing in mind the generally limited number of taxa and restricted morphological and molecular data analysed in some of these studies, as well as the use of different methods of phylogenetic and phenetic analysis, the results provide conflicting support for the generic status of Lutzia. With the exception of Mallampalli (1995), analyses based solely on morphological data 
support the generic status of Lutzia (Navarro et al. 2000; St John 2007; Harbach et al. 2012) by placing it outside the clade comprised of the subgenera of Culex (and also genera Deinocerites and Galindomyia, in studies where these were included). In stark contrast, analyses based on DNA sequence data (ITS1 and ITS2 rDNA and COI mtDNA) do not, placing Lutzia instead among species or subgenera of genus Culex (Miller et al. 1996; Deus 2009; Vesgueiro et al. 2011; Demari-Silva et al. 2011).

Our preliminary studies in connection with a broader study of culicinine relationships suggested that there is also conflict between adult and larval morphological data when analysed separately and in combination; thus, the present comparative study was undertaken to investigate in more detail whether the alternative positions of Lutzia are genuinely due to signal conflict or simply a lack of strong signal.

\section{Materials and methods}

\section{Morphology}

We added six species to the data set of 86 species analysed by Harbach et al. (2012): Culex (Culex) annulirostris Skuse, Cx. (Cux.) quinquefasciatus Say, Cx. (Culiciomyia) semibrunneus Edwards, Cx. (Eumelanomyia) insignis (Carter), Cx. (Lophoceraomyia) mammilifer (Leicester) and Cx. (Lop.) rubithoracis (Leicester) (see Harbach et al. 2012 for authorship of the other species mentioned herein, and all species included in the analyses). These species were added because the data were already available from another study and on the premise that they would strengthen support for the groups to which they belong. The full data set of 92 taxa thus comprised an ingroup of 87 species of Culicini and five outgroup species from other tribes of subfamily Culicinae: Culiseta (Culiseta) annulata (Culisetini), Mansonia (Mansonia) titillans (Mansoniini), Maorigoeldia argyropus (Sabethini), Orthopodomyia anopheloides (Orthopodomyiini) and Psorophora (Psorophora) ciliata (Aedini). The ingroup includes the type species of most generic-level taxa and the nominotypical species of all informal species groups and subgroups of subgenus Culex (Harbach 2011), except for the Coronator and Guiarti Groups. The 169 anatomical characters used by Harbach et al. (2012), fourthinstar larvae (76), pupae (15), adults (47), male genitalia (30) and habitat of immature stages (1), were coded for the six added species, using the protocols described in that paper, and these data are presented in Table 1. Missing data are indicated by "?", and characters that could not be scored due to absence of homologous structures ("dependent characters") are indicated by "-". Polymorphic characters are explicitly coded as exhibiting only those states observed. For analyses of the subset of the data comprising only the larval characters, subgenus Afroculex was excluded as it lacked all data from this life stage (the larval stage is unknown).

As previously (Harbach et al. 2012), the adjectival term "culicinine" is used to refer to species of tribe Culicini. Abbreviations used for the names of genera and subgenera follow Reinert (2009).

Specimens from the following collections were examined during the study: Natural History Museum (NHM), London, UK; National Museum of Natural History 
ลิธ

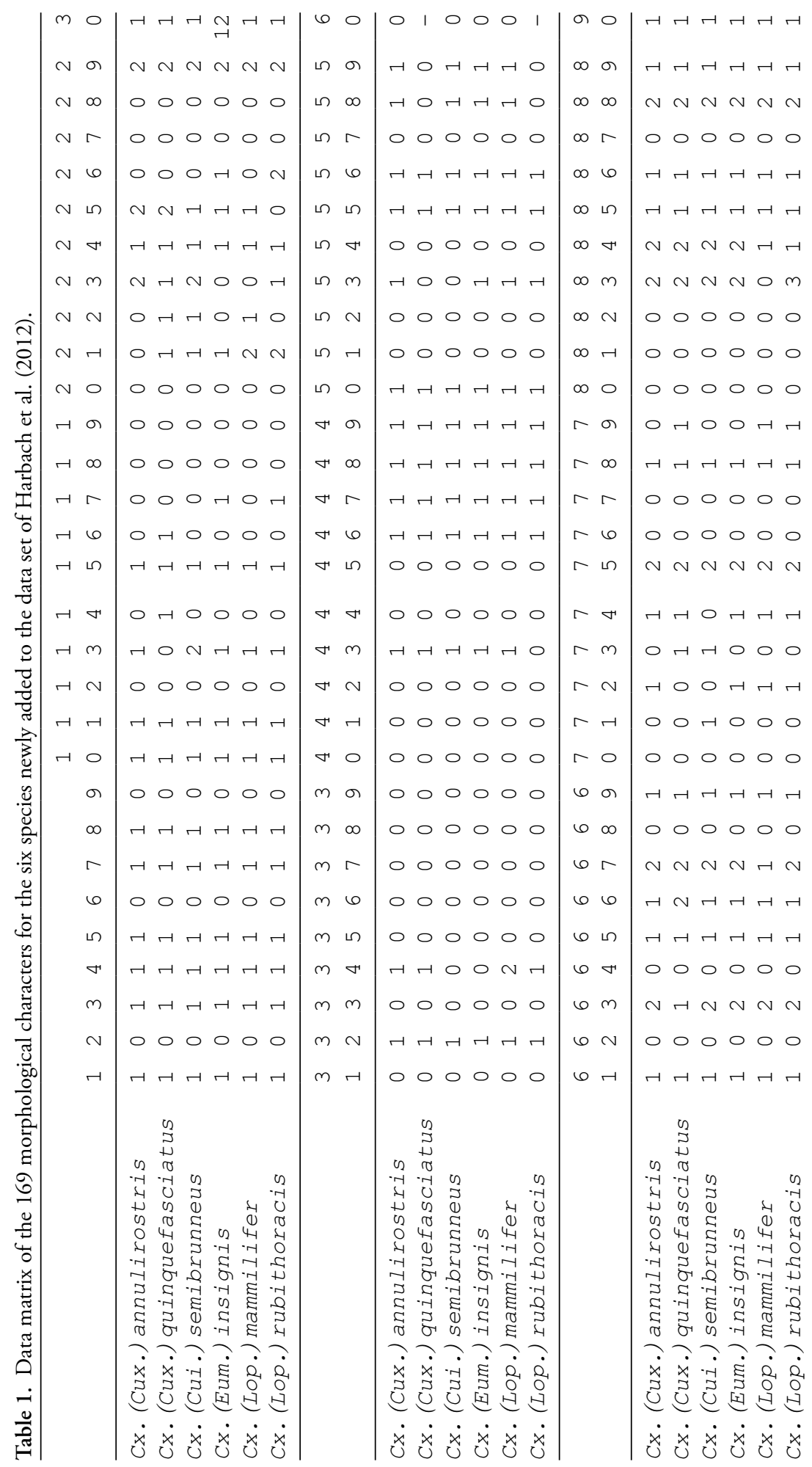




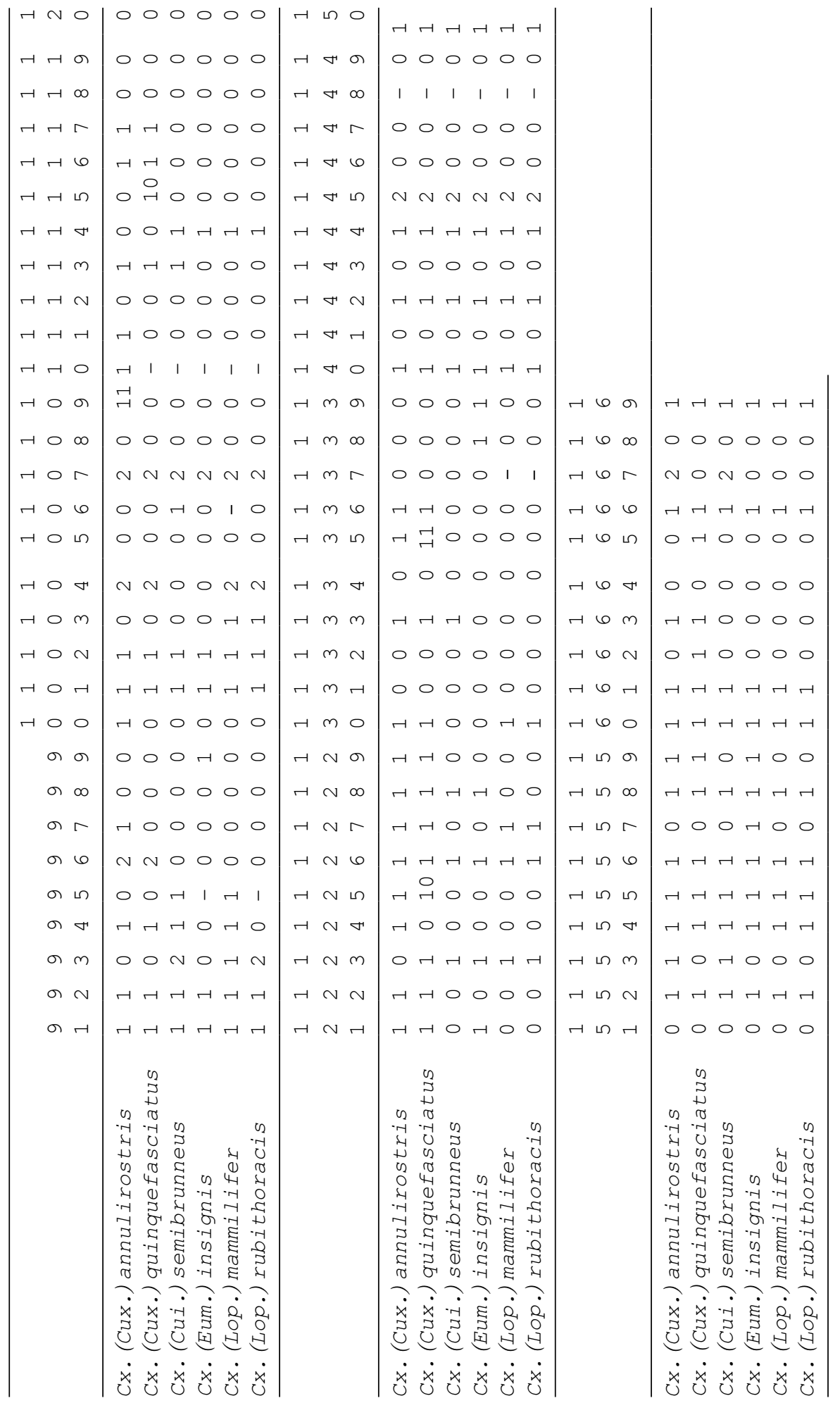


(USNM), Smithsonian Institution, Washington, DC, USA; and the Laboratoire de Taxonomie des Vecteurs, Centre IRD de Montpellier, France. Identification of specimens was confirmed from associated larval exuviae and dissected male genitalia.

\section{Phylogenetic analyses}

Phylogenetic analyses were carried out under the maximum parsimony (MP) criterion using implied weighting (IW) implemented with TNT version 1.1 (Willi Hennig Society Edition, August 2011) (Goloboff et al. 2008b). Tree searches were conducted using all four "New Technology" search options: sectorial searches, ratchet, tree drifting and tree fusing. For the ratchet, the up/downweighting probabilities were set to $5 \%$ and the number of replicates to 200 . The number of cycles of tree drifting was set to 50. All other search parameters were left at their default settings. Analyses were terminated once the most parsimonious (= fittest) cladogram(s) (MPCs) had been found 25 times. The maximum number of trees held was set to 10000 . Multistate characters were treated as unordered.

The application of Bremer support (Bremer 1994) to weighted data can lead to incorrect conclusions regarding group support (Goloboff et al. 2003). We therefore assessed clade support using symmetric resampling, as implemented in TNT, recording the frequency differences ("Groups present/Contradicted" or GC values; Goloboff et al. 2003). The GC values assess the difference between the absolute frequency with which a clade is found in the resampled matrices and that of the most frequent alternative topology in which the clade is not recovered. The GC values range from 100, where the clade is recovered in all resampled matrices, to -100 , where an alternative arrangement is found in all resampled matrices (Goloboff et al. 2003), and a value of zero indicates that levels of support and contradiction are equal. Due to time constraints, we calculated GC values using the "Traditional Search" options with 10000 replicates and the default change probability, with searches constrained to use only those groups found in the MPCs. The default setting, in which clades with GC $<1$ were collapsed in the support tree, was applied.

All cladograms were rooted between Culiseta and the remaining taxa. Cladograms were prepared and morphological character mappings investigated using WinClada ver. 1.00.08 (Nixon 1999-2002). For reasons of space, only selected cladograms are shown; those cladograms that are not shown, together with the various data sets, are available in the Dryad Digital Repository (Kitching et al. 2014).

\section{Results and discussion}

\section{Effects of the newly added taxa on morphological relationships}

We initially investigated the effects of the six added species on the preferred pattern of relationships found by Harbach et al. (2012). To determine the best value of the concavity constant, $K$, to use for subsequent analyses, we first undertook a series of 
analyses using $K$ values ranging from 4 to 20 (following Harbach et al. 2012). Again, only a single MPC (cladograms not shown) was recovered for each value of $K$ (but this time also including $K=4$, for which Harbach et al. 2012 found two MPCs) and a series of "plateaux of stability" were found involving $K$ values of $5-6,8-9,11-12$, $13-15,17-18$ and 19-20. One of these included the preferred $K$ value of 9 , and so we elected to use this value in all subsequent analyses for ease of comparison with the results of Harbach et al. (2012). The single MPC obtained (fit $=66.19509, \mathrm{CI}=0.15$, $\mathrm{RI}=0.55)$ is shown in Fig. 1 . The six added species are each placed with or in the groups expected based on the current internal classification of Culex (Harbach 2011). Culex (Cux.) quinquefasciatus is recovered as sister to $C x$. (Cux.) pipiens $+C x$. (Cux.) australicus (Group G of Harbach et al. 2012), and Cx. (Cux.) annulirostris is sister to a clade comprising $C x$. (Cux.) barraudi, Cx. (Cux.) edwardsi and $C x$. (Cux.) thalassius (Group B of Harbach et al. 2012). The remaining four newly added species, $C x$. (Cui.) semibrunneus, Cx. (Eum.) insignis, Cx. (Lop.) mammilifer and Cx. (Lop.) rubithoracis, are all included within Group L of Harbach et al. (2012). However, although subgenus Lophoceraomyia is still recovered as monophyletic, subgenus Culiciomyia is paraphyletic, with the newly coded $C x$. (Cui.) semibrunneus placed as sister to subgenus Acalleomyia plus the remaining two species of subgenus Culiciomyia, and subgenus Eumelanomyia is polyphyletic; the newly coded Cx. (Eum.) insignis is placed as sister to subgenus Lophoceraomyia rather than with Cx. (Eum.) inconspicuosus, which retains its sister-group relationship with subgenera Culiciomyia and Acalleomyia. Overall, the other changes wrought on the preferred $K=9$ hypothesis of Harbach et al. (2012: fig. 3) are relatively small, and in particular it should be noted that there was no effect on the placement of Lutzia as the sister group to Culex (including Deinocerites and Galindomyia). The largest differences concern the altered positions of $C x$. (Sir.) boninensis, which has moved to become sister to $C x$. (Cux.) annulirostris + Group B, and Group $\mathrm{K}$, which has moved from being sister to a terminal clade comprising subgenus Neoculex and Groups L and M, to a median position as sister to Groups F and G.

\section{Morphological data partition analyses}

Next, we undertook separate analyses of the adult (characters 93-169) and larval (characters 1-77) morphological data subsets to investigate whether the data from these two life stages yielded congruent patterns of relationship. A separate analysis of the pupal subset (characters 78-92) was not carried out because there were insufficient characters to provide meaningful resolution in the resultant MPCs.

Adult data. The single MPC (fit $=24.67439, \mathrm{CI}=0.18, \mathrm{RI}=0.66$ ) derived from analysis of the adult data only is shown in Fig. 2. The pattern differs from that obtained from analysis of the full data set in many ways. Of particular note is that Lutzia is no longer the sister-group of Culex. Instead, the first taxon to branch off within Culicini is $C x$. (Cux.) duttoni followed by $C x$. (Cux.) tarsalis. Lutzia is placed within the next clade to branch off but is not recovered as monophyletic. Instead, subgenus Metalutzia is sister to a clade comprised of four subgenera of Culex (Lasiosiphon, Afroculex, 


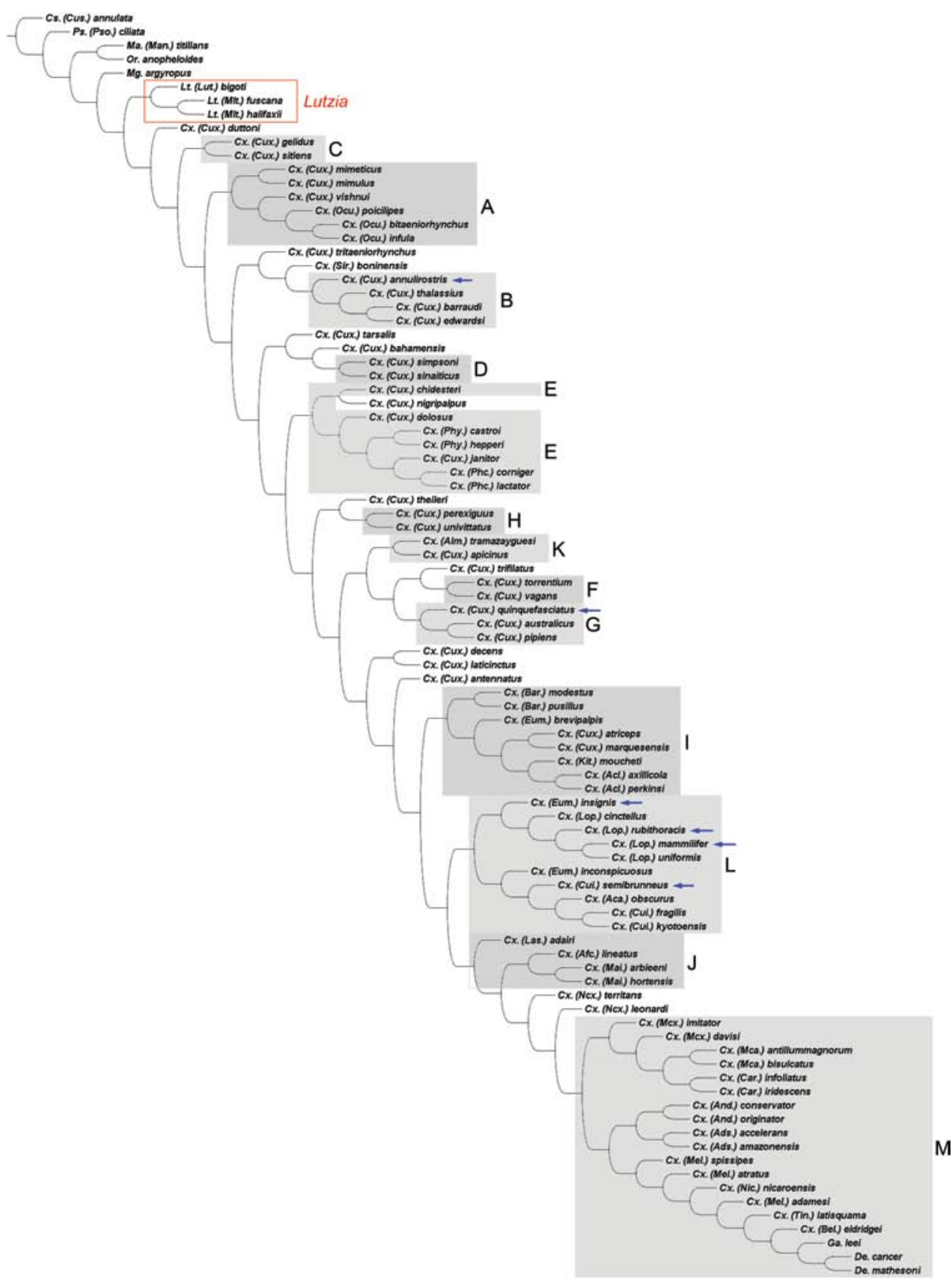

Fig. 1. The single most parsimonious cladogram obtained from implied weighting analysis of all characters (fit $=66.19509)$, showing the positions of the six newly added species (arrows). 


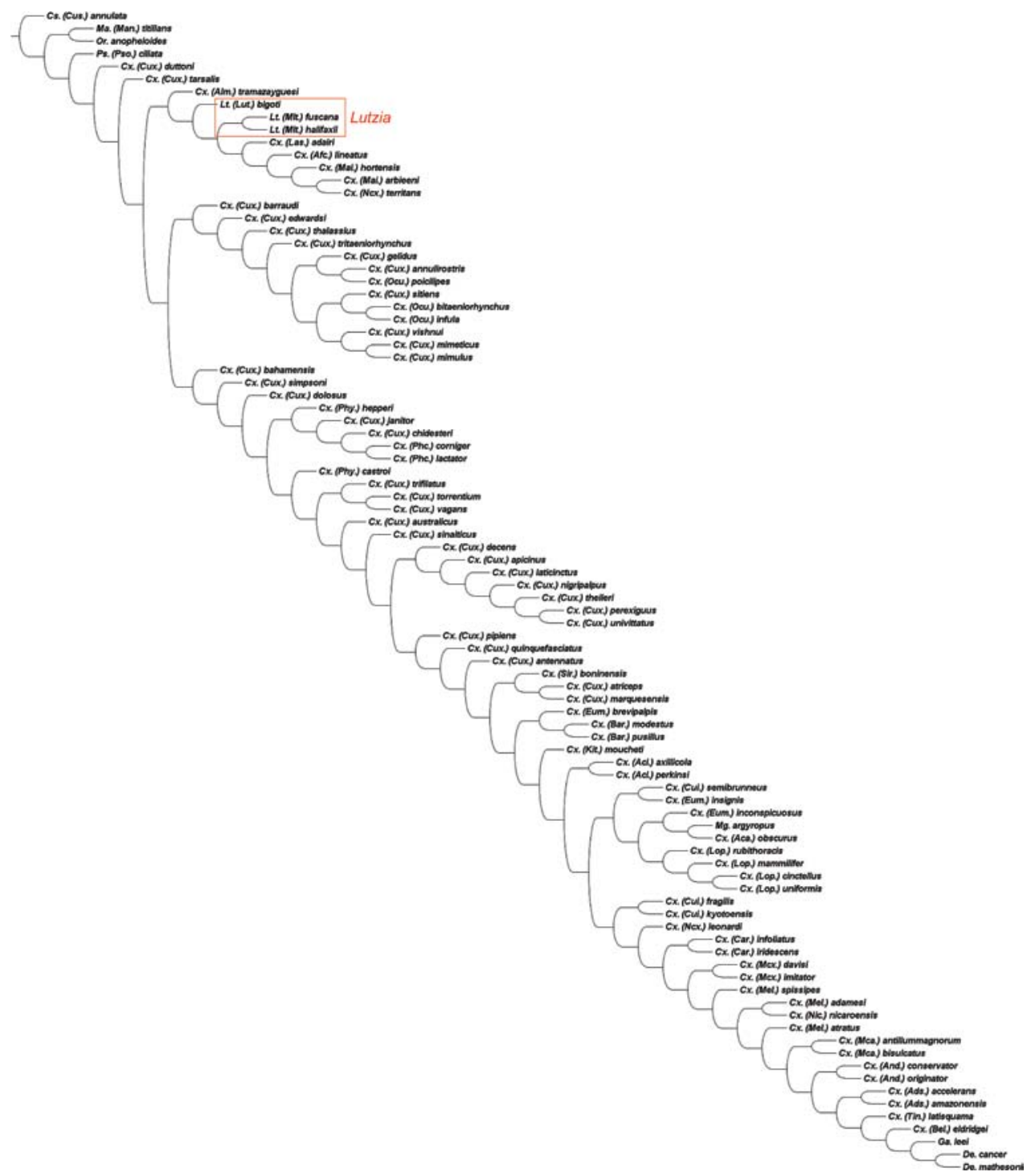

Fig. 2. The single most parsimonious cladogram obtained from implied weighting analysis of adult characters only (fit $=24.67439)$.

Maillotia and Neoculex), and this clade of seven taxa is sister to subgenus Lutzia, which is then followed by $C x$. (Allimanta).

Larval data. The single MPC (fit $=30.72456, \mathrm{CI}=0.15, \mathrm{RI}=0.54$ ) derived from analysis of the larval data only is shown in Fig. 3. The relationships of Lutzia are markedly different. Rather than being basal, it is now placed deep inside Culex, within one of two large clades, where it is sister to the outgroup genus Psorophora. These two genera are sister to a clade consisting of Culex subgenera Acalleomyia, Aedinus, Belkinomyia, Eumelanomyia, Oculeomyia, Tinolestes, three species of subgenus Culex (Cx. bahamensis 


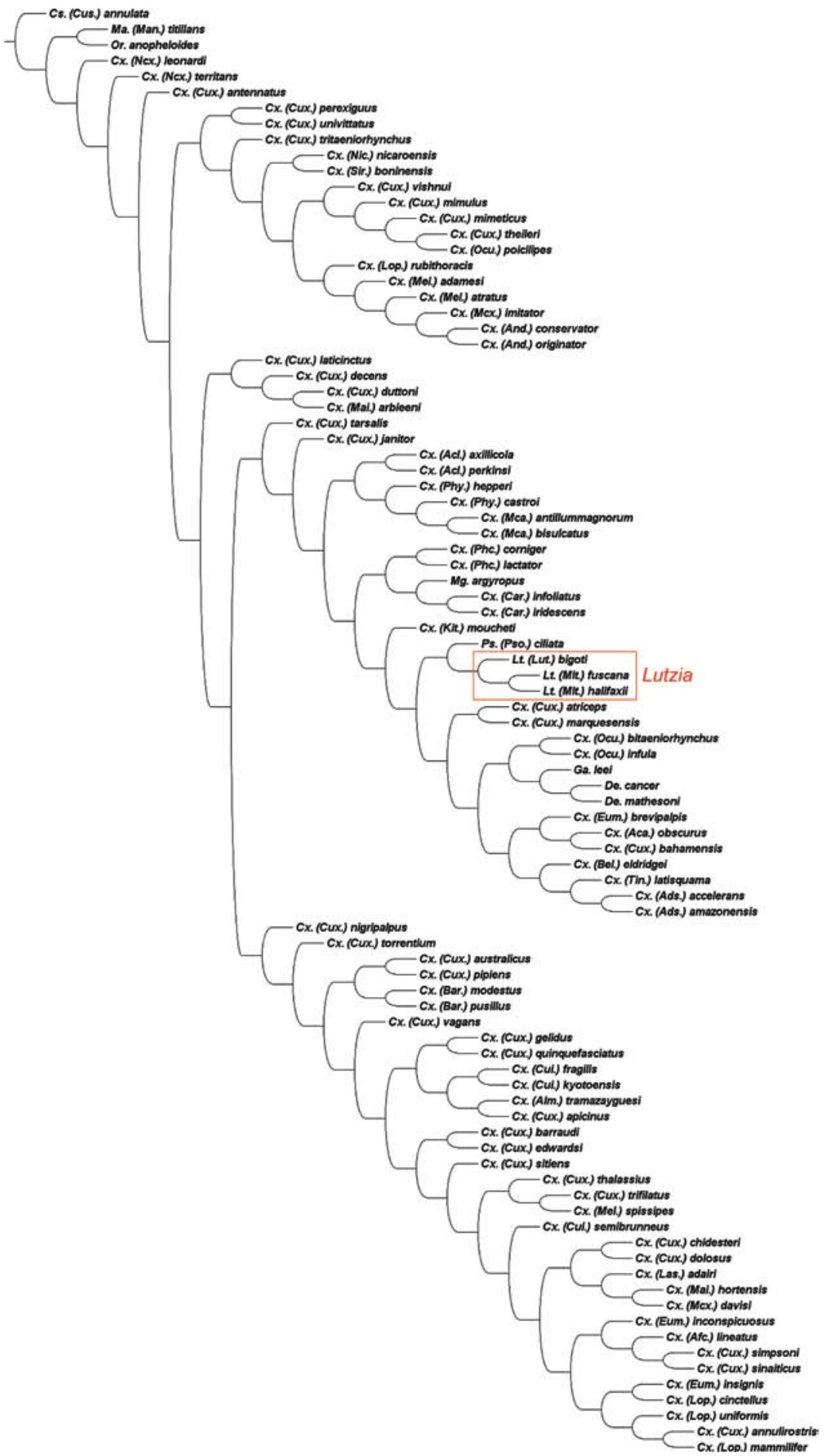

Fig. 3. The single most parsimonious cladogram obtained from implied weighting analysis of larval characters only (fit $=30.72456)$. 
and $C x$. atriceps $+C x$. marquesensis), and the genera Deinocerites and Galindomyia. The sister of this large clade is $C x$. (Kitzmilleria), and then a clade comprising $C x$. (Carrollia), $C x$. (Phenacomyia) and the outgroup genus Maorigoeldia. The sister to all these is then a clade comprising Culex subgenera Acallyntrum, Phytotelmatomyia and Micraedes.

Five larval characters unite Lutzia and Psorophora ciliata, of which four (characters 24:0, seta 2-P of larvae shorter than seta 1-P; 77:0, immature stages inhabit fresh-water ground pools; 82:1, seta 2-VI of pupae inserted anterior to anteromesad of seta 1-VI; and 120:1, prealar scales present in adults) are homoplastic, and also occur in support of other groups. However, on this cladogram (Fig. 3), the fifth character (2:1, lateral palatal brushes of larvae with about 40 thickened filaments) is optimized as synapomorphic for Lutzia and the outgroup species Ps. ciliata. The majority of culicinine larvae feed on suspended particulate matter and microorganisms and filter these from the water with numerous filamentous mouth brushes. As noted in the Introduction, Lutzia larvae are distinguished from larvae of other culicinine genera in having mouthparts modified for predation, with the lateral palatal brushes modified to aid in grasping prey. The lateral palatal brushes are similarly developed, though not as strongly, and have a similar function in larvae of Ps. ciliata, which are also predatory (Carpenter \& LaCasse 1955; Shalaby 1957; Belkin et al. 1970). Thus, the association of Lutzia and Psorophora when only larval data are considered would appear to be due largely to convergent feeding adaptations that are being misinterpreted here as synapomorphic.

Thus, neither the adult data nor the larval data independently support a sister-group relationship between Lutzia and the remaining Culicini. It is only when these data subsets are combined and analysed together that this relationship is recovered.

\section{Outgroup exclusion analyses}

Given the apparent convergence between Lutzia and Psorophora when only the larval data set is analysed, we ran a series of outgroup exclusion analyses on morphological data (all data, adult data only, larval data only) to test effects of removing each of these taxa in turn. Of the 15 possible sets of topologies, only those where there is a pattern of relationships of particular note are included with the discussion below.

When either Culiseta or Mansonia are removed, there is no effect on the position of Lutzia - it remains as sister to the remaining Culicini (cladograms not shown). When Maorigoeldia is excluded, Lutzia is still part of the first clade to branch off the ingroup but is paired with Culex subgenus Oculeomyia (cladogram not shown). In the pattern of relationships obtained from analysis of the full data (Fig. 1), although Lutzia was placed near to Psorophora, they were not sister taxa but separated by several branches, and so we did not expect that the position of Lutzia would change after exclusion of Psorophora. This, however, was not the case (Fig. 4; fit $=64.94990$ ). Lutzia is now nested deep inside Culicini as sister to $C x$. atriceps $+C x$. marquesensis. These are then sister to a clade comprising Culex subgenera Kitzmilleria and Acallyntrum, and all these are in turn sister to subgenus Eumelanomyia. Another major change in topology is the placement of the outgroup Maorigoeldia in the terminal large clade of Culicini as sister to Culex subgenus Carrollia. Similar unexpected changes in the topology occurred 


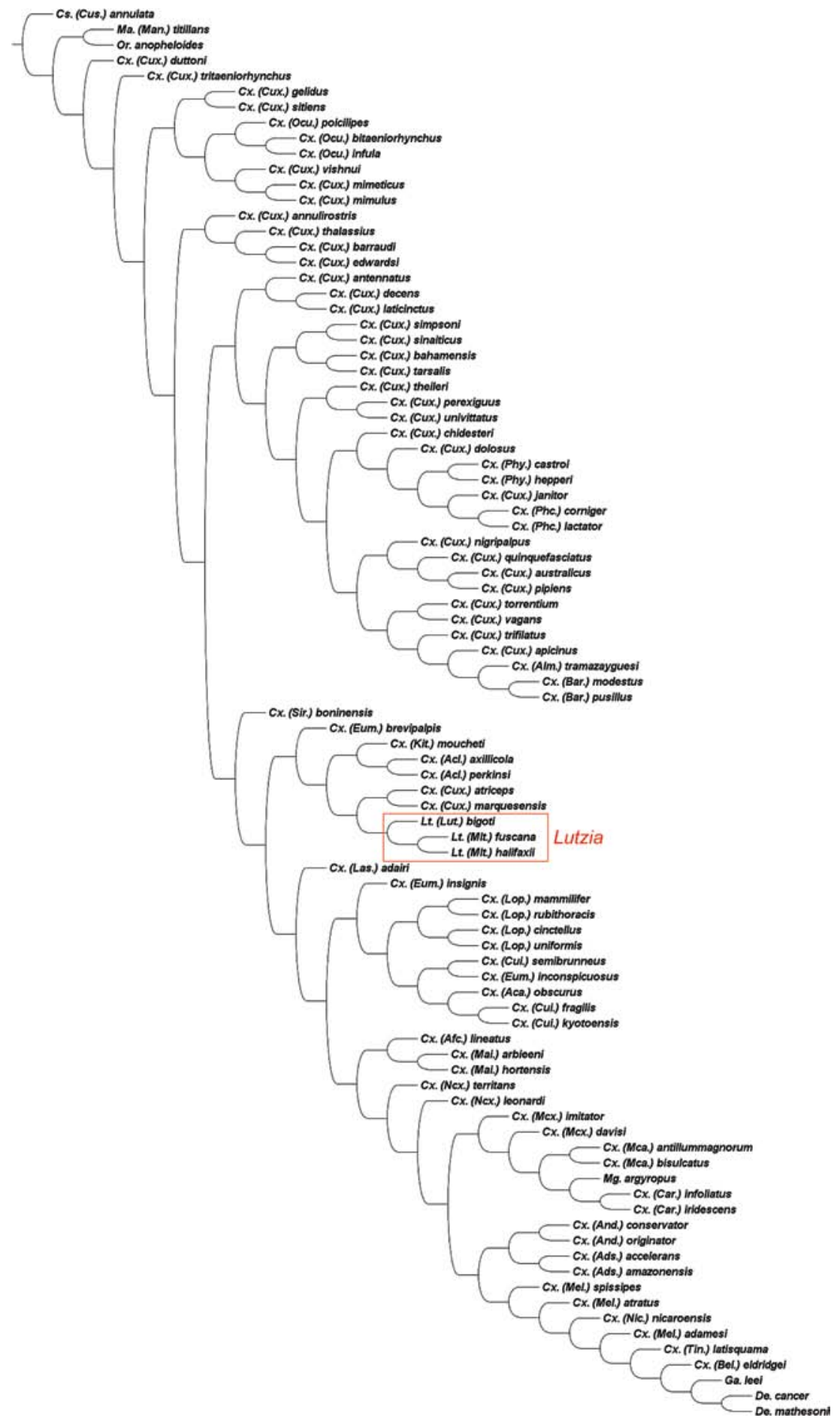

Fig. 4. The single most parsimonious cladogram obtained from implied weighting analysis of all characters, with outgroup Psorophora excluded (fit $=64.94990)$. 
when Orthopodomyia was excluded (Fig. 5; fit $=65.18498$ ). Kitzmilleria and Acallyntrum are now the first groups to branch off within Culicini and Lutzia is nested deep within the tribe as sister to subgenus Oculeomyia. The sister to Lutzia + Oculeomyia is Cx. atriceps $+C x$. marquesensis, then $C x$. (Cux.) duttoni (moved up from a basal position in Fig. 1), Cx. (Cux.) bahamensis and a clade of another three species of subgenus Culex.

When only the adult morphological data are analysed, Lutzia is paraphyletic and placed in a clade with Culex subgenera Afroculex, Allimanta, Lasiosiphon, Maillotia and Neoculex (Fig. 2). This pattern of relationships is also largely recovered when Culiseta, Mansonia, Maorigoeldia or Psorophora is excluded, although in the last case Culex subgenus Allimanta is placed as sister to all other Culicini (cladograms not shown). When Orthopodomyia is excluded, the members of this group are still placed close to one another but now as a paraphyletic grade (cladogram not shown). Thus, when only adult data are considered, it seems that the relationships of Lutzia are reasonably robust to outgroup exclusion.

Analysis of the larval data only placed Lutzia in a markedly different position, as sister to outgroup Psorophora in a clade that also included the outgroup Maorigoeldia, ingroup genera Deinocerites and Galindomyia, Culex subgenera Acalleomyia, Aedinus, Belkinomyia, Carrollia, Eumelanomyia, Kitzmilleria, Oculeomyia, Phenacomyia and Tinolestes, and three species of subgenus Culex $(C x$. bahamensis and $C x$. atriceps $+C x$. marquesensis) (Fig. 3). This clade, with the same internal relationships, was also recovered when either Culiseta or Orthopodomyia was excluded (cladograms not shown) and the sister-group relationship between Lutzia and Psorophora was also recovered in all other analyses (except, of course, that in which Psorophora was excluded). When Maorigoeldia was removed, Lutzia + Psorophora was placed as sister to subgenus Kitzmilleria, and these three as sister to Carrollia + Phenacomyia (cladogram not shown). The remaining taxa listed above are now positioned more distally elsewhere on the cladogram so that the sister to the Phenacomyia-Lutzia clade is now the clade comprised of subgenera Acallyntrum, Phytotelmatomyia and Micraedes. These are then followed by $C x$. (Cux.) janitor, and this whole group is sister to all other Culicini.

A similar pattern of relationships is recovered when Psorophora is excluded (cladogram not shown). However, the outgroup Maorigoeldia is now sister to Lutzia, followed by Carrollia, then Phenacomyia, and Kitzmilleria is now sister to Acallyntrum in a clade together with Micraedes and Phytotelmatomyia. These two groups form a clade that is sister to $C x$. (Cux.) janitor, and the whole is sister to all other Culicini. The inclusion of Maorigoeldia within Culicini was not expected, nor was its pairing with Lutzia, which is based on four homoplastic characters: characters 24:0, 26:0, 67:3 and $99: 1$. The apomorphic state of the first character (thoracic seta 2-P equal in length to seta 1-P) is found in all sampled taxa except Lutzia + Maorigoeldia, the other three outgroup taxa and $C x$. $(\mathrm{Ocu}$.$) infula, in which it is reversed to the plesiomorphic state$ (thoracic seta 2-P $<0.8$ times the length of seta 1-P). Character 26 (development of thoracic seta 3-P) is highly homoplastic (steps $=20, \mathrm{CI}=0.10, \mathrm{RI}=0.47)$ and state 0 occurs in many other species, as does character 99 (presence/absence of scales on the first antennal flagellomere; steps $=22, \mathrm{CI}=0.04, \mathrm{RI}=0.46$ ). State 3 of character 67 


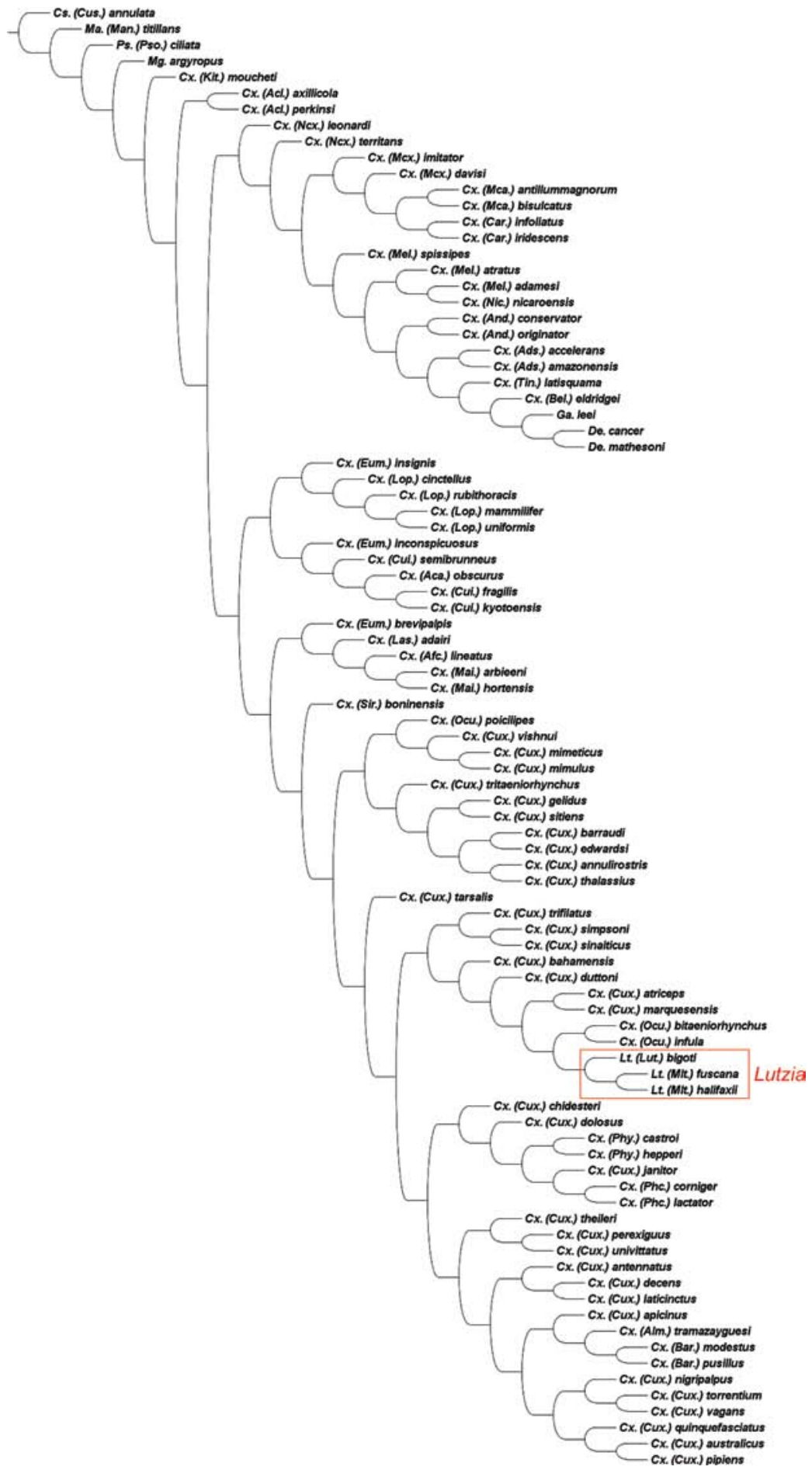

Fig. 5. The single most parsimonious cladogram obtained from implied weighting analysis of all characters, with outgroup Orthopodomyia excluded (fit = 65.18498). 
appears independently six times scattered across the cladogram but is the only trait that offers potentially reasonable support to the Lutzia + Maorigoeldia clade. Character 67 refers to the number of pairs of seta 1-S on the siphon, state 3 being the case where there are seven or more pairs of setae. Harbach et al. (2012) noted that this condition normally occurs in species with either a very long siphon or when seta 1-S is in a single zigzag or irregular double posterior row, but did not comment on the condition in either Lutzia or Maorigoeldia, although seta 1-S is developed similarly in the two genera (cf., figs 124, Lutzia, and 362, Maorigoeldia, of Belkin 1962b).

When Mansonia was excluded, analysis of the larval data produced a different pattern of relationships (strict consensus tree of 3 MPCs not shown). Although Lutzia remains paired with Psorophora, and is still part of a clade that also contains ingroup genera Deinocerites and Galindomyia, Culex subgenera Acalleomyia, Aedinus, Belkinomyia, Eumelanomyia, Kitzmilleria, Oculeomyia, Tinolestes and three species of subgenus Culex (Cx. bahamensis and $C x$. atriceps $+C x$. marquesensis), the internal relationships of this clade are rather changed. Furthermore, the sister group to this large clade in adult data-only analyses, comprising the outgroup genus Maorigoeldia and Culex subgenera Carrollia and Phenacomyia, is moved to a much more basal position.

Overall, therefore, outgroup selection seems to have an impact on the phylogenetic patterns recovered within Culicini, and can result in markedly different placements for Lutzia. Adding another, more distant outgroup from another subfamily (e.g. Anophelinae) could help stabilise the root of the cladograms but then might introduce new issues with regard to homology determination and missing data effects. But nevertheless a question remains as to how much of this apparent incongruity is meaningful, i.e. how strongly supported are these conflicting groups?

\section{Clade support for groups derived from analysis of morphology}

One issue with implied weighting as implemented in TNT is the high precision of its calculations, a result of which is that rarely more than one MPC is recovered (Goloboff 2013). Consequently, it is important that a conservative approach to group acceptance is adopted (Goloboff et al. 2008a). Hence, we assessed clade support using symmetrical resampling and recorded the frequency differences (GC values; Goloboff et al. 2003).

Using the default setting in TNT, we collapsed all groups with GC values $<1$. When applied to the single MPC derived from analysis of the full morphological data set (Fig. 1), the result was a collapse of relationships into a very large basal polytomy that comprises 28 clades or species, one of which is Lutzia (Fig. 6). When groups with GC $<1$ were collapsed in the MPC derived from analysis of the adult only data (Fig. 2), an even larger, 42-component polytomy resulted (cladogram not shown) in which, not unsurprisingly, Lutzia is not recovered as monophyletic. The collapse of the MPC derived from analysis of the larval only data (Fig. 3) was the most extreme, with the basal polytomy now comprised of 53 clades or species (cladogram not shown). One of these is Psorophora + Lutzia, but support for the relationship is weak with GC $=7$.

When the groups with $\mathrm{GC}<1$ were collapsed in the MPCs derived from each of the 15 outgroup exclusion analyses, the results showed essentially the same patterns that 


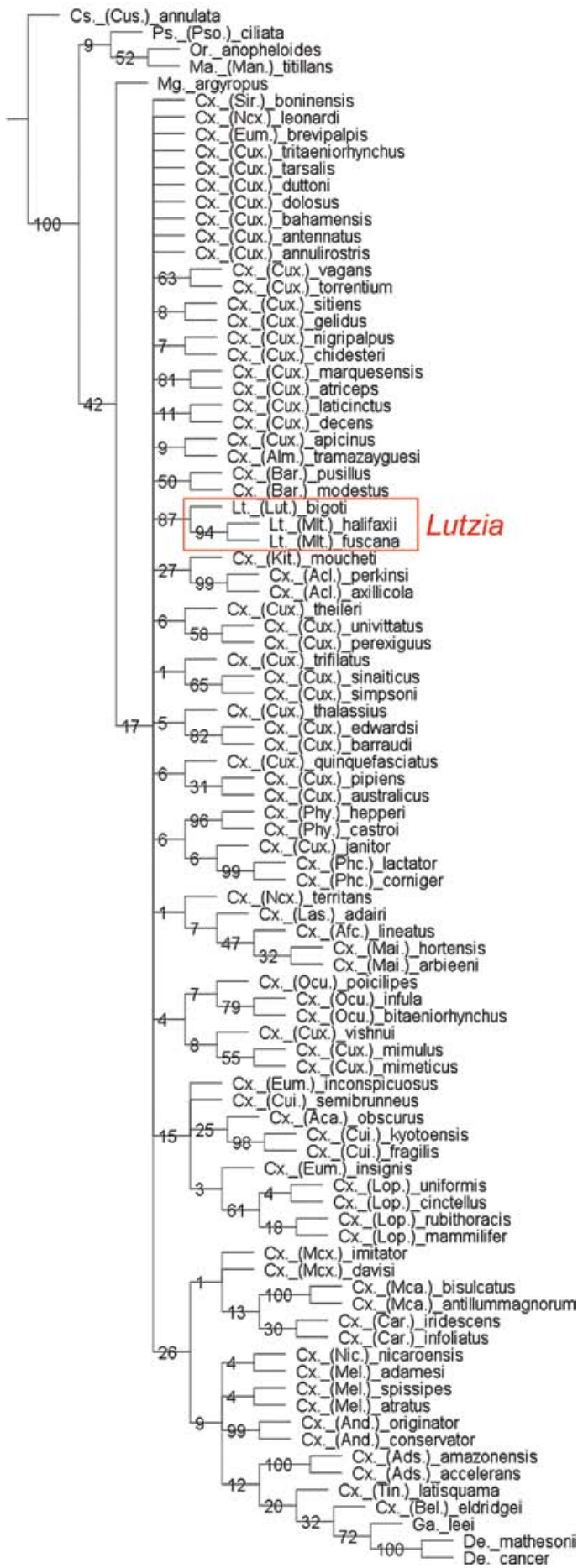

Fig. 6. The cladogram obtained from implied weighting analysis of all characters, after all branches with GC values $<1$ are collapsed (GC values for remaining clades are show on the branches). 
were recovered when the full data set was analysed (cladograms not shown). Thus, in the cladograms derived from the analysis of all characters, Lutzia was recovered as monophyletic in a large basal polytomy; when only adult characters were analysed, the two subgenera of Lutzia were recovered as separate elements of the large basal polytomy; and when only larval characters were analysed, a weakly supported clade comprised of Psorophora + Lutzia (GC =3-16) was recovered in the basal polytomy, with the obvious exception of when Psorophora was excluded, whence Lutzia formed an independent clade in the basal polytomy. Thus, it seems that the topological inconsistencies in the position of Lutzia (and many of the other taxa as well) in the MPCs derived from all analyses of the various morphological data sets and subsets (Figs 1-5 and cladograms not shown) are spurious. The placement of Lutzia is not well-supported in any of the cladograms and we must conclude that there is no strong evidence from morphology to place Lutzia either as the sister-group of all other Culicini or within genus Culex. Consequently, we refrain from selecting a preferred topology from those discussed above, and we do not present a figure with character optimizations mapped onto such a cladogram because we consider that the situation is too ambiguous for character optimizations to be meaningful.

All four previous phylogenetic analyses of culicinine mosquitoes based on morphological data (Navarro \& Liria 2000; St John 2007; Harbach et al. 2012; Laurito \& Almirón 2013) placed Lutzia as sister to the remaining taxa of Culicini. However, the last of these (i.e. Laurito \& Almirón 2013) used Lutzia to root their cladogram and thus this position is uninformative with regard to whether or not Lutzia is sister to Culex. Furthermore, if, as above, we accept only those groups with $\mathrm{GC} \geq 10$, then most of the branches in their tree collapse and Lutzia would be part of a large basal polytomy. Navarro \& Liria (2000) included an Ochlerotatus species as a more distant outgroup and did recover Lutzia as sister to Culex (including Deinocerites). However, most of their clades too were poorly supported, with bootstrap (BS) values $<60 \%$. If we accept that a bootstrap value of $70 \%$ or more is indicative of reasonable to strong support (Alfaro \& Holder 2006, and references therein), and then collapse all of their branches with a bootstrap value of $<70 \%$, then only two clades survive: subgenus Carrollia and a pair of its species, Cx. (Car.) bihaicola Dyar \& Núñez Tovar $+C x$. (Car.) rausseoi Cova García, Sutil Oramas \& Pulido, F.

St John (2007) analysed 64 morphological characters using MP and Bayesian inference (BI) techniques, and in all cases Lutzia was placed as sister to all other culicinines. However, under equal weighting (St John 2007: fig. 3) none of the branches along the main stem of the MPC (including the sister clade to Lutzia) had bootstrap values over $50 \%$, and Bremer support values (BS; Bremer 1994) were also very low, generally 1 , occasionally 0 or 2 . Furthermore, the partitioned Bremer support values derived from the adult, male genitalia, larval and pupal data subsets often showed considerable contradictory support, suggesting low GC values. Application of successive approximations character weighting raised the bootstrap value of the sister clade of Lutzia to 76\% (St John 2007: fig. 4). However, this is spurious because, as Goloboff et al. (2003) pointed out, when characters are differentially weighted group support can be over- or under-estimated due to inequalities in the way the characters are sampled (hence, our 
use of GC values, which were proposed specifically by Goloboff et al. (2003) to circumvent this problem). Likewise, the main stem branches of the tree derived from the BI analysis (St John 2007: fig. 6) were all <0.9. One consensus that has emerged is that only groups with Bayesian posterior probabilities (BPP) $>0.9$, or even 0.95 , should be regarded as "well supported" (Wilcox et al., 2002; Rodrigues et al., 2010), and if this criterion is applied, then all of these branches must also be collapsed. Thus, the basal position of Lutzia is at best only very weakly supported.

Harbach et al. (2012) did not include support values for the clades in their preferred MPCs and so the strength of support for the placement of Lutzia as sister to all other Culicini cannot be evaluated (but see below).

In contrast, previous analyses based on DNA sequence data alone have agreed in placing Lutzia among species or subgenera of genus Culex. Deus (2009) used ITS1 and ITS2 to investigate the phylogeny of subgenus Culex, and included Lutzia (Metalutzia) tigripes (de Grandpre \& de Charmoy) as one of a number of outgroup taxa (Deinocerites cancer and species of subgenera Melanoconion $(n=3)$, Micraedes $(n=1)$ and Neoculex $(n=1))$. When ITS1 alone was analysed using MP, Lutzia was recovered as the strongly supported sister to subgenus Culex (with subgenus Phenacomyia included within it) (BS $=100 \%$ ) (Deus 2009: fig. 2.14). Analysis of either ITS2 alone (Deus 2009: fig 2.16) or together with ITS1 (Deus 2009: fig. 2.12) placed Lutzia in a cluster with the other outgroup taxa, although without support. However, the position of Lutzia is dependent on how the cladograms were rooted and Deus was inconsistent, rooting the ITS1 cladogram on Melanoconion pilosus (Dyar \& Knab) but rooting the other two between the outgroup taxa as a whole and subgenus Culex (including Phenacomyia). The absence of any outgroup taxon beyond Culicini makes an unequivocal positioning of the root impossible. Of course, this was actually irrelevant to the study of Deus, which was focused on the internal relationships of subgenus Culex.

Vesgueiro et al. (2011) also used ITS2 and, in contrast, found strong support for Lutzia to be placed within Culex (including Phenacomyia) (BS = 90-100\%). However, their analysis was a phenetic neighbour-joining approach and thus not comparable with phylogenetic approaches. A phylogenetic reanalysis of their data might prove enlightening.

The results of our separate analyses of adult and larval data were also equivocal, appearing to support different patterns of relationships with regard to the position of Lutzia. Again, most of the conflicting clades are poorly supported and once these are removed from consideration, most of the conflict disappears (but so does much of the resolution). This and the results of the molecular studies noted above would suggest, given our current state of knowledge, that neither morphology nor mitochondrial or ribosomal sequence alone have sufficiently strong signal to resolve the position of Lutzia. For COI sequence, this is perhaps not surprising. If Lutzia were indeed the sister group of Culex, then this split would have occurred during the Cretaceous more than 100 Mya (based on divergence time estimates and credibility intervals calculated by Reidenbach et al. 2009; for comparison, Wiegmann et al. 2001 found that Culicidae diverged from Chaoboridae about $140 \mathrm{Mya}$ ). This is more than enough time for the sequence to become saturated and the phylogenetic signal obscured. For morphology, 
the situation is less obvious. Most morphological features are undoubtedly influenced by a number of genes, are highly heritable and have been the target of natural selection. Unfortunately, major differences in morphological features may be the result of rapid radiation rather than ancient divergence, which has been hypothesised for other groups of insects (Grimaldi \& Engel 2005). Thus, although morphological distinctions are easily observed and characterised, they may provide misleading clues about evolutionary relationships.

Harbach et al. (2012) did not map characters onto their preferred cladogram, but in our equivalent Fig. 1, the 10 characters that support Culicini excluding Lutzia are 7:1; $17: 0 ; 23: 1 ; 24: 1 ; 66: 2 ; 67: 2 ; 84: 2 ; 99: 0 ; 135: 1$ and 163:1. Seven of these are highly homoplastic with between 14 and 28 steps (character consistency index, CI $=0.21-$ 0.05 ), but three deserve closer consideration. All are unambiguously optimised as being developed in Culex, but then undergo further transformations. Character 7:1 (larval antenna length $0.75-1.00$ times length head capsule; 8 steps, $\mathrm{CI}=0.25$, character retention index, $\mathrm{RI}=0.76)$ is independently reversed five times: $\mathrm{Cx}$. $(\mathrm{Ocu}$. $)$ infula; Phytotelmatomyia + Phenacomyia + Cx. (Cux.) janitor, Kitzmilleria + Acallyntrum $+C x$. (Cux.) atriceps + Cx. (Cux.) marquesensis; Carrollia + Micraedes; and Deinocerites + Galindomyia. The second character, 24:1 (thoracic seta 2-P approximately equal in length to seta 1-P; 2 steps, $\mathrm{CI}=0.50, \mathrm{RI}=0.87)$, reverses only in $C x$. $($ Ocu. $)$ infula. Finally, character 163:1 (ventral arm of opisthophallic sclerite present; 4 steps, CI = $0.25, \mathrm{RI}=0.92)$ is reversed in subgenus Allimanta and in the clade above Cx. (Cux.) antennatus, then redeveloped in $C x$. (Cux.) atriceps $+C x$. (Cux.) marquesensis.

\section{Conclusions}

It would appear that no data set analysed in any study conducted to date has adequate signal to place Lutzia unequivocally with regard to other taxa in Culicini. Perhaps only more data from nuclear DNA sequence, from genes with appropriate rates of change or transcriptomics stand a chance of solving this problem. Reidenbach et al. (2009) used DNA sequence data from six nuclear genes and 80 morphological characters to investigate the suprageneric relationships among 25 mosquito genera. The BPP values for most clades in their preferred phylogram (Reidenbach et al. 2009: fig. 3) exceed the 0.95 threshold, suggesting the possibility that these genes may be the right ones to resolve intra-tribal relationships of mosquitoes.

From a practical classification viewpoint, the studies conducted thus far also offer no compelling evidence to support the recognition of Lutzia as either a genus or as a subgenus of Culex. However, when questioning the generic-level status of culicinine taxa, consideration must be given to the following points:

(1) Lutzia, along with species of Deinocerites and subgenera Melanoconion, Micraedes and Neoculex, was placed outside subgenus Culex in the ITS1 and ITS2 MP phylogenies of Deus (2009), although whether as sister to subgenus Culex (with subgenus Phenacomyia subordinate within it) or as part of an outgroup cluster depended upon whether the ITS1 and ITS2 sequences were analysed separately or together. 
(2) Genus Deinocerites (and Galindomyia when included) has consistently fallen within genus Culex in phylogenies based on both morphological and DNA sequence data (Mallampalli 1995; Navarro \& Liria 2000; St John 2007; Deus 2009; Harbach et al. 2012).

(3) Fifteen of the 26 subgenera of genus Culex (Acalleomyia, Aedinus, Anoedioporpa, Carrollia, Culex, Culiciomyia, Eumelanomyia, Lophoceraomyia, Maillotia, Melanoconion, Micraedes, Microculex, Neoculex, Oculeomyia and Tinolestes) were originally described as genera based on morphological distinctions. As noted in the Introduction, morphological and molecular data support the monophyly of all of the generic-level groups of Culicini except subgenera Culex, Eumelanomyia and Neoculex. The question to be answered is whether the phylogenetic relatedness of groups based on morphological data should be ranked as genera, subgenera or at some other level of classification.

In view of this information, it is obvious that the generic and subgeneric classification of Culicini is problematic. If Lutzia, Deinocerites and Galindomyia are retained as genera, then a number of taxa currently recognised as subgenera of Culex should probably be granted generic status. On the other hand, if Lutzia is reduced to subgeneric status within Culex, then Deinocerites and Galindomyia should be also. So, at this point the question of whether current evidence supports generic or subgeneric status for Lutzia is largely irrelevant in view of the questionable relationships and taxonomic ranks of many of the culicinine taxa.

\section{Acknowledgements}

We are grateful to Philippe Boussès and Didier Fontenille (Institut de Recherche pour le Développement (IRD), Montpellier, France) and James Pecor (Walter Reed Biosystematics Unit (WRBU), Smithsonian Institution, Washington, DC, USA) for assistance with loans of specimens; to Erica McAlister and Theresa Howard (Natural History Museum (NHM), London) for curatorial assistance. We are indebted to the following individuals for providing specimens used in this study: Jeronimo Alencar (Instituto Oswaldo Cruz, Rio de Janeiro, Brazil); Shelley Cook (NHM); Seth Irish (London School of Hygiene and Tropical Medicine, London); and Yvonne Linton (WRBU). We gratefully acknowledge the sponsorship of the Willi Hennig Society for making TNT ver. 1.1 freely available. The study was carried out as part of a research project funded by the Leverhulme Trust, London (grant no. F/00 696/S).

\section{References}

Alfaro, M.E. \& Holder, M.T. (2006) The posterior and the prior in Bayesian phylogenetics. Annual Review of Ecology, Evolution and Systematics 37: 19-42.

Belkin, J.N. (1962a) The mosquitoes of the South Pacific (Diptera, Culicidae) Volume 1. University of California Press, Berkeley, CA, xii +608 pp. 
Belkin, J.N. (1962b) The mosquitoes of the South Pacific (Diptera, Culicidae) Volume 2. University of California Press, Berkeley, CA, 412 pls.

Belkin, J.N., Heinemann, S.J. \& Page, W.A. (1970) The Culicidae of Jamaica (Mosquito studies. XXI). Contributions of the American Entomological Institute 6: 1-458.

Bremer, K. (1994) Branch support and tree stability. Cladistics 10: 295-304.

Carpenter, S.J. \& LaCasse, W.J. (1955) Mosquitoes of North America (north of Mexico). University of California Press, Berkeley, CA, vii + 360 pp., 127 pls.

Demari-Silva, B., Vesgueiro, F.T., Sallum, M.A.M. \& Marrelli, M.T. (2011) Taxonomic and phylogenetic relationships between species of the genus Culex (Diptera: Culicidae) from Brazil inferred from the cytochrome $c$ oxidase I mitochondrial gene. Journal of Medical Entomology 48: 272-279.

Deus, S. (2009) Phylogeny of selected species of subgenus Culex mosquitoes (Diptera: Culicidae) from the U.S., Puerto Rico, and Guatemala, based on nucleotide sequences from three genes. PhD thesis, Department of Microbiology, Immunology and Pathology, Colorado State University, Fort Collins, CO: vi + $160 \mathrm{pp}$.

Edwards, F.W. (1932) Genera Insectorum. Diptera, Fam. Culicidae. Fascicle 194. Desmet-Verteneuil, Brussels, 258 pp., 4 pls.

Goloboff, P.A. (2013) Extended implied weighting. Cladistics, in press, DOI: 10.1111/cla.12047.

Goloboff, P.A., Farris, J., Källersjö, M., Oxelmann, B., Ramírez, M. \& Szumik, C. (2003) Improvements to resampling measures of group support. Cladistics 19: 324-332.

Goloboff, P.A., Carpenter, J., Arias, S. \& Miranda, D. (2008a) Weighting against homoplasy improves phylogenetic analysis of morphological data sets. Cladistics 24: 758-773.

Goloboff, P.A., Farris, J.S. \& Nixon, K.C. (2008b) TNT, a free program for phylogenetic analysis. Cladistics 24: 774-786.

Grimaldi, D. \& Engel, M.S. (2005) Evolution of the Insects. Cambridge University Press, Cambridge, England: $772 \mathrm{pp}$.

Harbach, R.E. (2011) Classification within the cosmopolitan genus Culex (Diptera: Culicidae): The foundation for molecular systematics and phylogenetic research. Acta Tropica 120: 1-14.

Harbach, R.E., Kitching, I.J., Culverwell, C.L., Dubois, J. \& Linton, Y.-M. (2012) Phylogeny of mosquitoes of tribe Culicini (Diptera: Culicidae) based on morphological diversity. Zoologica Scripta 41: 499-514 + 8 online figures and 2 appendices.

Isoe, J. (2000) Comparative analysis of the vitellogenin genes of the Culicidae. $\mathrm{PhD}$ thesis, Faculty of the Interdisciplinary Program in Insect Science, University of Arizona, Tucson, AZ: 203 pp.

Juthayothin, T. (2004) Molecular phylogenetic study of culicine mosquitoes using the mitochondrial cytochrome oxidase I gene and the relationships with mosquito-borne flaviviruses. MSc thesis, Faculty of Graduate Studies, Mahidol University, Bangkok: iv + 258 pp.

Kitching, I.J., Culverwell, C.L. \& Harbach, R.E. (2014) Data from: The phylogenetic conundrum of Lutzia (Diptera: Culicidae: Culicini): a cautionary account of conflict and support. Available online from the Dryad Digital Repository at http://doi.org/10.5061/dryad.5s790.

Laurito, M. \& Almirón, W.R. (2013) Phylogenetic relationships of Culex (Culex) species (Diptera, Culicidae) from Argentina based on morphological characters. Zootaxa 3652: 117-155.

Mallampalli, V.L. (1995) Phylogenetic relationships among taxa in the genus Culex, and the phylogenetic relationships of vectors of New World alphaviruses in the subgenus Melanoconion (Diptera: Culicidae). PhD thesis, Department of Entomology, University of Maryland, College Park, MD: xii + 250 pp.

Miller, B.R., Crabtree, M.B. \& Savage, H.M. (1996) Phylogeny of fourteen Culex mosquito species, including the Culex pipiens complex, inferred from the internal transcribed spacers of ribosomal DNA. Insect Molecular Biology 5: 93-107.

Navarro, J.C. \& Liria, J. (2000) Phylogenetic relationships among eighteen Neotropical Culicini species. Journal of the American Mosquito Control Association 16: 75-85.

Nixon, K.C. (1999-2002). WinClada version 1.00.08. Published by the author. Ithaca, NY, available online at http://www.cladistics.org. 
Reidenbach, K.R., Cook, S., Bertone, M.A., Harbach, R.E., Wiegmann, B.M. \& Besansky, N.J. (2009) Phylogenetic analysis and temporal diversification of mosquitoes (Diptera: Culicidae) based on nuclear genes and morphology. BMC Evolutionary Biology 9: 298.

Reinert, J.F. (2009) List of abbreviations for currently valid generic-level taxa in family Culicidae (Diptera). European Mosquito Bulletin 27: 68-76.

Rodrigues, S.G., Nunes, M.R.T., Casseb, S.M.M., Prazeres, A.S.C., Rodrigues, D.S.G., Silva, M.O., Cruz, A.C.R., Tavares-Neto, J.C. \& Vasconcelos, P.F.C. (2010) Molecular virology of Saint Louis encephalitis virus in the Brazilian Amazon: genetic divergence and dispersal. Journal of General Virology 91: 2420-2427.

Shalaby, A.M. (1957) The mouth parts of the larval instars of Psorophora ciliata (Fabricius). Bulletin de la Société entomologique d'Égypte 41: 429-455.

St John, O. (2007) Phylogeny of the genus Culex (Diptera: Culicidae). MRes thesis, Department of Life Sciences, Imperial College, London: ii +20 pp.

Tanaka, K. (2003) Studies on the pupal mosquitoes of Japan (9). Genus Lutzia, with establishment of two new subgenera, Metalutzia and Insulalutzia (Diptera, Culicidae). Japanese Journal of Systematic Entomology 9: 159-169.

Tanaka, K.,,Mizusawa, K. \& Saugstad, E.S. (1979) A revision of the adult and larval mosquitoes of Japan (including the Ryukyu Archipelago and the Ogasawara Islands) and Korea (Diptera: Culicidae). Contributions of the American Entomological Institute 16: vii + 1-987.

Theobald, F.V. (1903) A monograph of the Culicidae or mosquitoes. Volume 3. British Museum (Natural History), London: xvii + 359 pp., 17 pls.

Vesgueiro, F.T., Demari-Silva, B., dos Santos Malafronte, R., Sallum, M.A.M. \& Marrelli, M.T. (2011) Intragenomic variation in the second internal transcribed spacer of the ribosomal DNA of species of the genera Culex and Lutzia (Diptera: Culicidae). Memórias do Instituto Oswaldo Cruz 106: 1-8.

Wiegmann, B.M., Trautwein, M.D., Winkler, I.S., Barr, N.B., Kim, J.-W., Lambkin, C., Bertone, M.A., Cassel, B.K., Bayless, K.M., Heimberg, A.M, Wheeler, B.M., Peterson, K.J., Pape, T., Sinclair, B.J, Skevington, J.H., Blagoderov, V., Caravas, J., Kutty, S.N., Schmidt-Ott, U., Kampmeier, G.E., Thompson, F.C., Grimaldi, D.A., Beckenbach, A.T., Courtney, G.W., Friedrich, M., Meier, R. \& Yeates, D.K. (2011) Episodic radiations in the fly tree of life. Proceedings of the National Academy of Sciences 108: 5690-5695 + online supporting information with 4 figures and 4 tables.

Wilcox, T.P., Zwickl, D.J., Heath, T.A. \& Hillis, D.M. (2002) Phylogenetic relationships of the dwarf boas and a comparison of Bayesian and bootstrap measures of phylogenetic support. Molecular Phylogenetics and Evolution 25: 361-371. 